\title{
The impact of serine protease HtrA in apoptosis, intestinal immune responses and extra-intestinal histopathology during Campylobacter jejuni infection of infant mice
}

Markus M Heimesaat ${ }^{1 *}$, André Fischer ${ }^{1}$, Marie Alutis ${ }^{1}$, Ursula Grundmann ${ }^{1}$, Manja Boehm², Nicole Tegtmeyer ${ }^{2}$, Ulf B Göbel ${ }^{1}$, Anja A Kühl ${ }^{3}$, Stefan Bereswill ${ }^{1+}$ and Steffen Backert ${ }^{2+}$

\begin{abstract}
Background: Campylobacter jejuni has emerged as a leading cause of bacterial enterocolitis. The serine protease HtrA has been shown to be a pivotal, novel C. jejuni virulence factor involved in cell invasion and transmigration across polarised epithelial cells in vitro. However, the functional relevance of the $h t r A$ gene for the interaction of $C$. jejuni with the host immune system in the infant mouse infection model has not been investigated so far.

Results: Here we studied the role of C. jejuni htrA during infection of 3-weeks-old infant mice. Immediately after

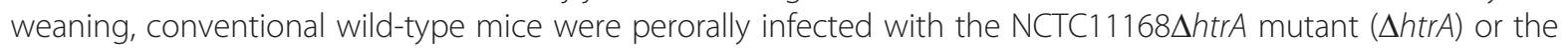
parental wild-type strain. Approximately one third of infected infant mice suffered from bloody diarrhea until day 7 post infection (p.i.), whereas colonic histopathological changes were rather moderate but comparable between the two strains. Interestingly, parental, but not $\triangle$ htrA mutant infected mice, displayed a multifold increase of apoptotic cells in the colonic mucosa at day 7 p.i., which was paralleled by higher colonic levels of pro-inflammatory cytokines such as TNF- $\alpha$ and IFN- $\gamma$ and the matrix-degrading enzyme matrixmetalloproteinase-2 (MMP-2). Furthermore, higher numbers of proliferating cells could be observed in the colon of $\Delta h t r A$ infected mice as compared to the parental wild-type strain. Remarkably, as early as 7 days p.i. infant mice also exhibited inflammatory changes in extra-intestinal compartments such as liver, kidneys and lungs, which were less distinct in kidneys and lungs following $\Delta h t r A$ versus parental strain infection. However, live C. jejuni bacteria could not be found in these organs, suggesting the induction of systemic effects during intestinal infection.
\end{abstract}

Conclusion: Upon C. jejuni $\Delta$ htrA strain infection of infant mice, intestinal and extra-intestinal pro-inflammatory immune responses were ameliorated in the infant mouse model system. Future studies will shed further light onto the molecular mechanisms of host-pathogen interactions.

Keywords: Cell invasion, Conventional infant mice, Ulcerative enterocolitis, Innate immunity, Host-pathogeninteraction, Apoptosis, Extra-intestinal immune responses, Hepatic, Renal, Pulmonal histopathology

\footnotetext{
* Correspondence: markus.heimesaat@charite.de

${ }^{\dagger}$ Equal contributors

'Department of Microbiology and Hygiene, Charité - University Medicine Berlin,

Campus Benjamin Franklin, Hindenburgdamm 27, D-12203 Berlin, Germany

Full list of author information is available at the end of the article
} 


\section{Background}

Camplylobacter jejuni displays a major infectious agent of foodborne bacterial enterocolitis of men with increasing prevalence in developed as well as developing countries $[1,2]$. Severity of campylobacteriosis varies from mild disease to acute symptoms such as abdominal cramps, fever, myalgia, and watery to bloody diarrhea [3]. Patients suffering from acute disease display crypt abscesses, ulcerations and colonic infiltration with pro-inflammatory immune cell populations [4-6]. Whereas the vast majority of $C$. jejuni infections is normally self-limiting in humans, post-infectious sequelae such as Guillain-Barré syndrome, Miller Fisher syndrome, Reiter's syndrome and reactive polyarthritis might arise in rare cases [3,7]. An important prerequisite for $C$. jejuni causing disease is its ability to adhere and invade intestinal epithelial cells [8]. A plethora of bacterial outer membrane proteins such as JlpA, CadF, FlpA, PEB1 among others has been shown to be involved in adhesion to epithelial cells [9-13], whereas CadF can induce the activation of small Rho GTPases, Rac1 and Cdc42, which exert invasive properties in vitro [13-16] and in human ex vivo biopsies [17]. We and others have recently shown that the $C$. jejuni serine protease and chaperone HtrA (high temperature requirement A) displays a novel virulence factor [18-21]. Whereas HtrA family members were considered in the past to strictly act intracellularly in the bacteria, we recently discovered that HtrA is actively secreted into the extracellular environment where it cleaves cell surface adhesion proteins and tumor-suppressor E-cadherin [21-23]. In vitro infection experiments with $C$. jejuni revealed that secreted HtrA is capable of opening cell-to-cell-junctions in the epithelium by cleaving-off the $90 \mathrm{kDa}$ extracellular domain of Ecadherin $[21,22]$. Furthermore, htrA gene deletion has been shown to result in defective E-cadherin shedding and compromised transmigration of $C$. jejuni across polarized epithelial cells in vitro [21].

The studies of molecular mechanisms of pathogenhost-interactions causing $C$. jejuni induced disease have been hampered by a lack of suitable in vivo models given that the host-specific composition of the microbiota determines the physiological colonization resistance against $C$. jejuni $[24,25]$. Whereas conventionally colonized adult (>8-weeks-old) mice expel the pathogen within a few days post infection, gnotobiotic wild-type mice and mice recolonized with human microbiota were readily colonized by C. jejuni [24]. However, classical clinical symptoms of human campylobacteriosis such as bloody diarrhea were missing in these murine infection models [24]. In contrast 3 -weeks-old infant mice are highly susceptible to C. jejuni infection and develop self-limiting bloody diarrhea within one week [25-30]. After resolving enterocolitis within another 7-10 days, infant mice were asymptomatic long-term $C$. jejuni carriers exhibiting distinct pro- inflammatory immune responses in intestinal as well as extra-intestinal locations such as liver, lungs, and kidneys characterized by influx of predominantly $\mathrm{T}$ (and less distinctly B) lymphocytes after more than 3 months p.i. [25,31]. In the present study, we applied the infant mouse model to investigate the functional relevance of the $h t r A$ gene in $C$. jejuni infection in vivo. Furthermore we studied potential extraintestinal inflammatory sequelae in the early course of C. jejuni induced disease.

\section{Results}

Intestinal colonization and clinical symptoms in infant mice following infection with wild-type and htrA mutant C. jejuni

Immediately after weaning conventional 3-weeks-old infant mice were perorally infected with approximately $10^{9}$ colony forming units (CFU) of either the $C$. jejuni knockout mutant NCTC11168::htrA $(\Delta h t r A)$ or the parental wild-type (WT) strain each harvested in the stationary phase on two consecutive days (day 0 and 1 ). Control analyses demonstrated that equal amounts of $C$. jejuni protein were infected per sample and the HtrA protein is not expressed in the $\Delta h t r A$ mutant as expected (Figure 1A). Seven days post infection (p.i.) less than half of parental and $\Delta h t r A$ strain infected mice harboured the pathogen in the large intestine $(8.3 \%$ and $46.2 \%$, respectively) with relatively low pathogenic loads between $10^{3}$ and $10^{7} \mathrm{CFU}$ per $g$ luminal content, whereas in the proximal and distal small intestinal tract either $C$. jejuni strain was virtually undetectable (Figure 1B). In addition, approximately one third of mice developed clinical symptoms of $C$. jejuni induced acute enterocolitis until day 7 p.i., as indicated by $33.3 \%$ and $38.5 \%$ positive cases of bloody diarrhea in parental and $\Delta h t r A$ strain infected mice, respectively (Figure 2A). We next assessed gradual histopathological changes in the mucosa and lamina propria of hematoxylin and eosin (H\&E) stained colonic paraffin sections. Seven days following infection with either strain mice displayed comparable histopathological scores and exhibited rather mild to moderate colonic histopathological changes such as single to mild scattered cell infiltrates within the colonic mucosa and submucosa, mild epithelial hyperplasia and starting loss of goblet cells (Figure 2B).

\section{C. jejuni HtrA aggravates intestinal apoptosis and immune responses}

Given that apoptosis is a commonly used diagnostic marker in the histopathological evaluation and grading of intestinal disease [24] and a key feature of C. jejuni induced ulcerative enterocolitis [25], we next quantitatively assessed apoptotic cells applying in situ immunohistochemical stainings of colonic paraffin sections. Seven days following parental, but not $\Delta h t r A$ strain infection, 


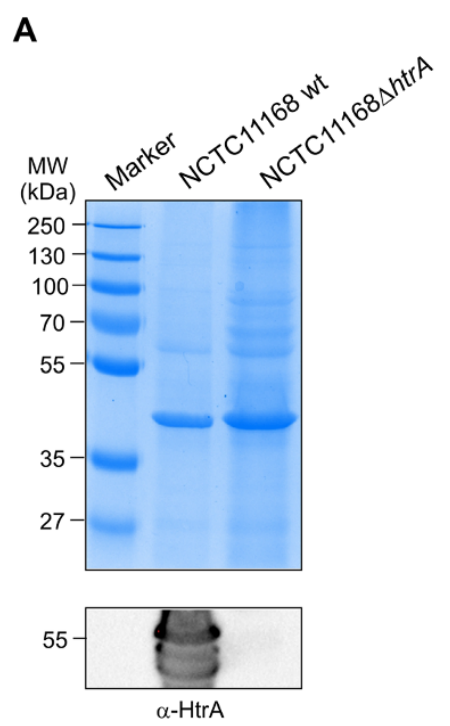

B

C. jejuni NCTC11168

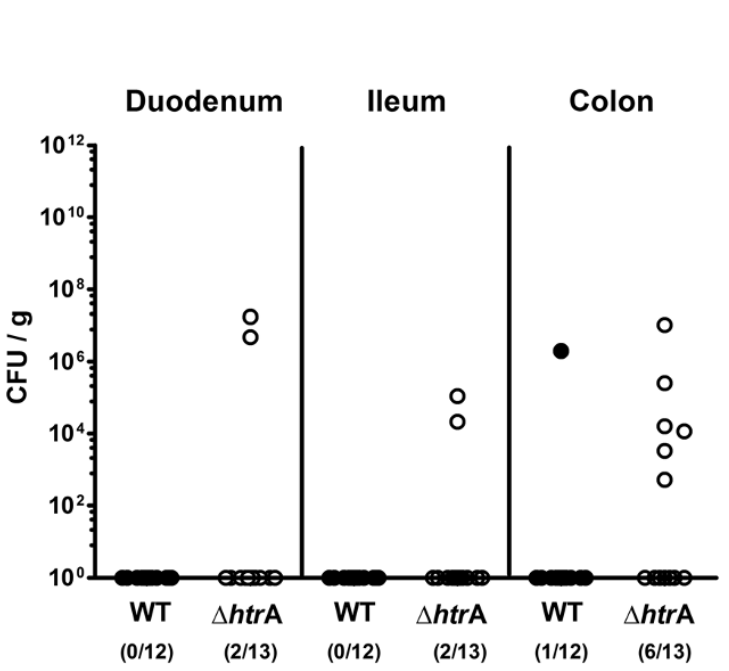

Figure 1 Influence of HtrA on C. jejuni colonization along the intestinal tract of infant mice. (A) Loading control (Coomassie blue staining) of proteins from total cell lysates (top) and Western blot for the expression of HtrA in the two C. jejuni strains used in this infection study.

(B) Immediately after weaning, conventional infant wild-type mice were perorally infected with C. jejuni NCTC11168 (WT, closed circles) or the mutant strain NCTC11168 $h$ trA ( $\Delta$ htrA, open circles). The pathogenic loads in distinct compartments of the intestinal tract were determined by quantification of live C. jejuni in luminal samples taken from duodenum, ileum, and colon at day 7 following infection by cultural analysis (CFU, colony forming units). Medians (black bars) are indicated and numbers of mice harbouring the respective C. jejuni strain out of the total numbers of analyzed animals are given in parentheses. Data shown were pooled from three independent experiments.

\section{A}

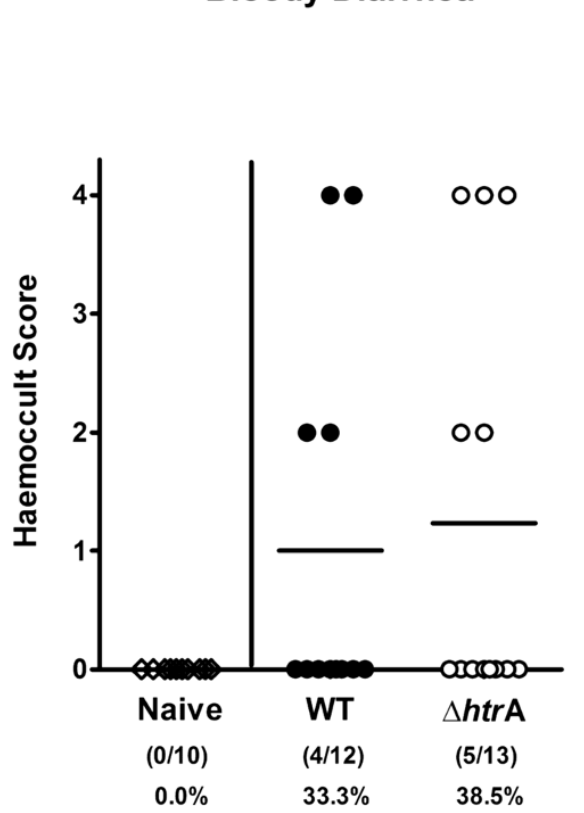

B

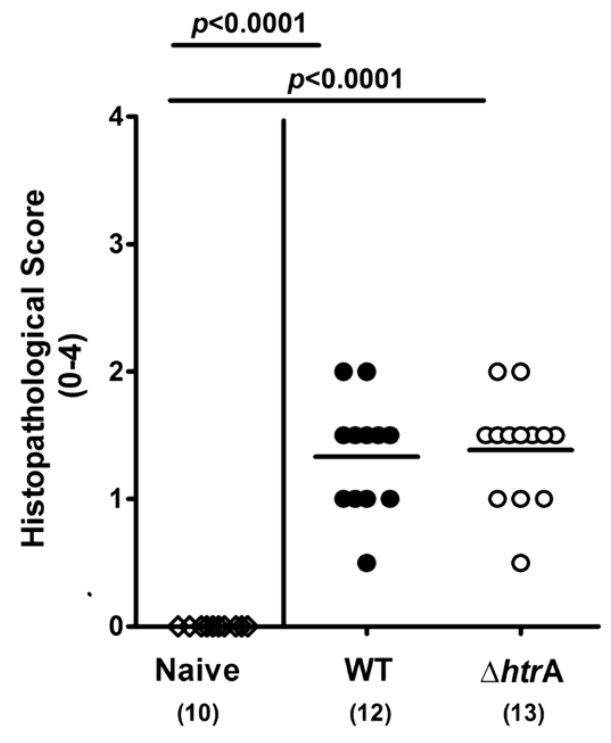

Figure 2 Role of HtrA in clinical symptoms and colonic histopathology in C. jejuni infected infant mice. Immediately after weaning, infant

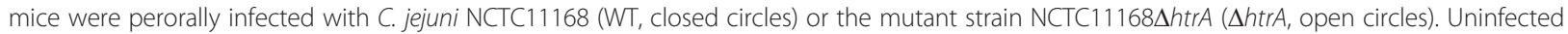
animals (Naïve; open diamonds) served as negative controls. Seven days following $C$. jejuni infection (A) occurrence of blood in fecal samples and (B) histopathological changes in H\&E stained colonic paraffin sections were assessed by applying standardized clinical and histophathological scoring systems, respectively. Means (black bars) and numbers of analyzed animals (in parentheses) or absolute and relative (in \%) numbers of positive samples out of the total number are indicated. Data shown were pooled from three independent experiments. 
infant mice displayed more than two fold higher colonic caspase3-positive apoptotic cell numbers as compared to naïve controls ( $\mathrm{p}<0.05$ vs. naïve; $\mathrm{p}<0.0001$ vs. $\Delta$ htr $A$ strain; Figure $3 \mathrm{~A})$. Furthermore, $\Delta h t r A$ strain infected mice exhibited more colonic Ki67-positive proliferating cells as compared to parental strain infected and naïve controls ( $\mathrm{p}<0.0005$ and $\mathrm{p}<0.001$, respectively; Figure $3 \mathrm{~B}$ ) indicative for upregulated regenerative epithelial function during immunopathology. Given that recruitment of immune cells is a hallmark of human campylobacteriosis [4,5] we next quantitatively assessed $\mathrm{T}$ cell populations in the large intestines of $C$. jejuni infected mice. Seven days following $\Delta h t r A$ strain infection, infant mice displayed a trend towards lower colonic CD3-positive T lymphocyte numbers as compared to parental strain infected control animals (not significant due to high SD in either group; Figure 3C), whereas Foxp3-positive regulatory $\mathrm{T}$ cells (Tregs) increased comparably in the colonic mucosa and lamina propria of mice upon peroral infection with either strain (Figure 3D).

\section{C. jejuni HtrA is necessary for the induction of TNF-a, IFN- $\gamma$ and matrixmetalloproteinase-2}

We next determined $C$. jejuni induced pro-inflammatory cytokine responses in colonic ex vivo biopsies. Colonic TNF- $\alpha$ protein and IFN- $\gamma$ mRNA expression levels increased almost two-fold 7 days following parental, but not $\Delta h \operatorname{tr} A$ infection $(\mathrm{p}<0.01$ and $\mathrm{p}<0.05$, respectively; Figure 4) further underlining the role of HtrA in aggravating $C$. jeuni mediated inflammation.

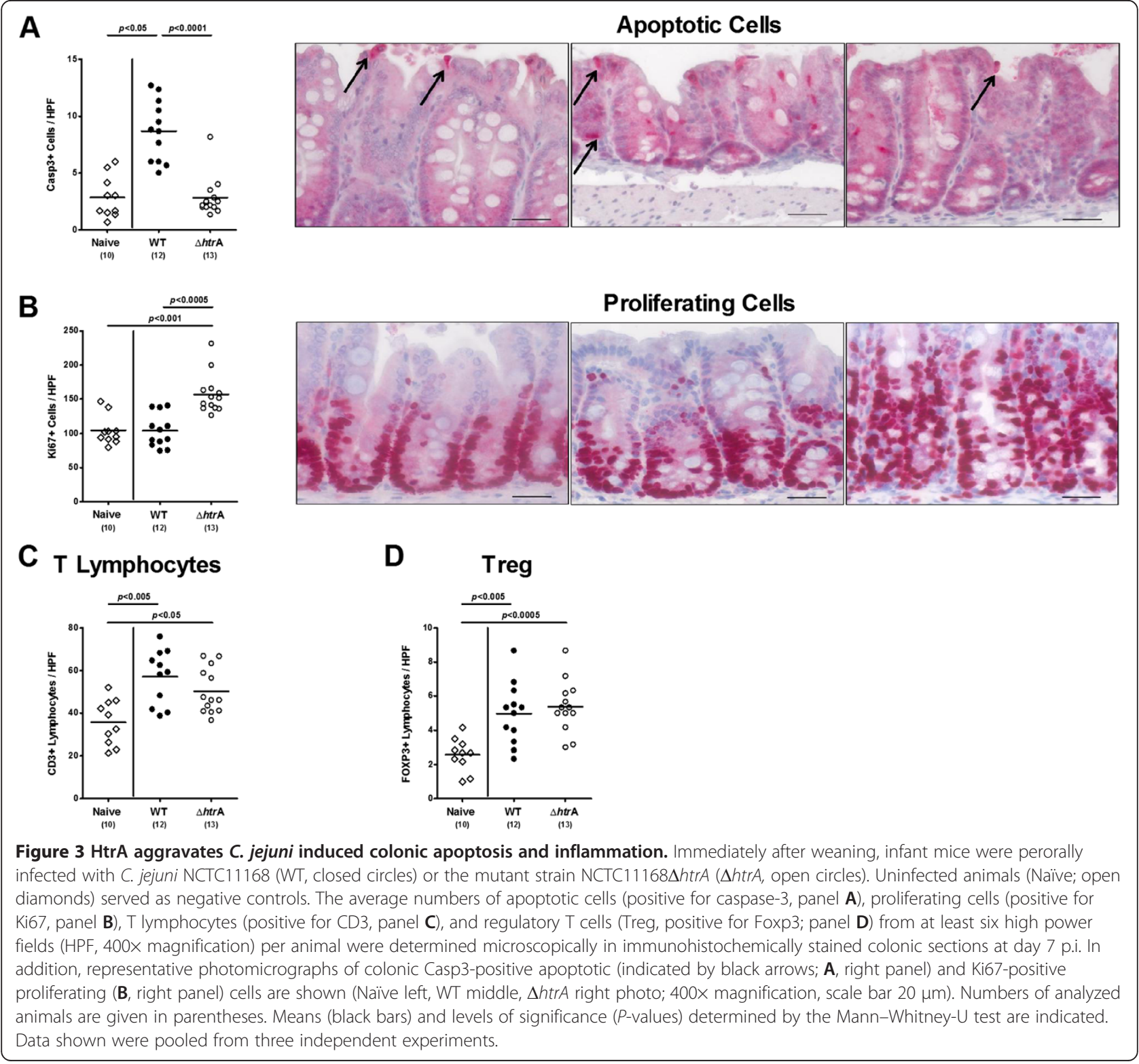




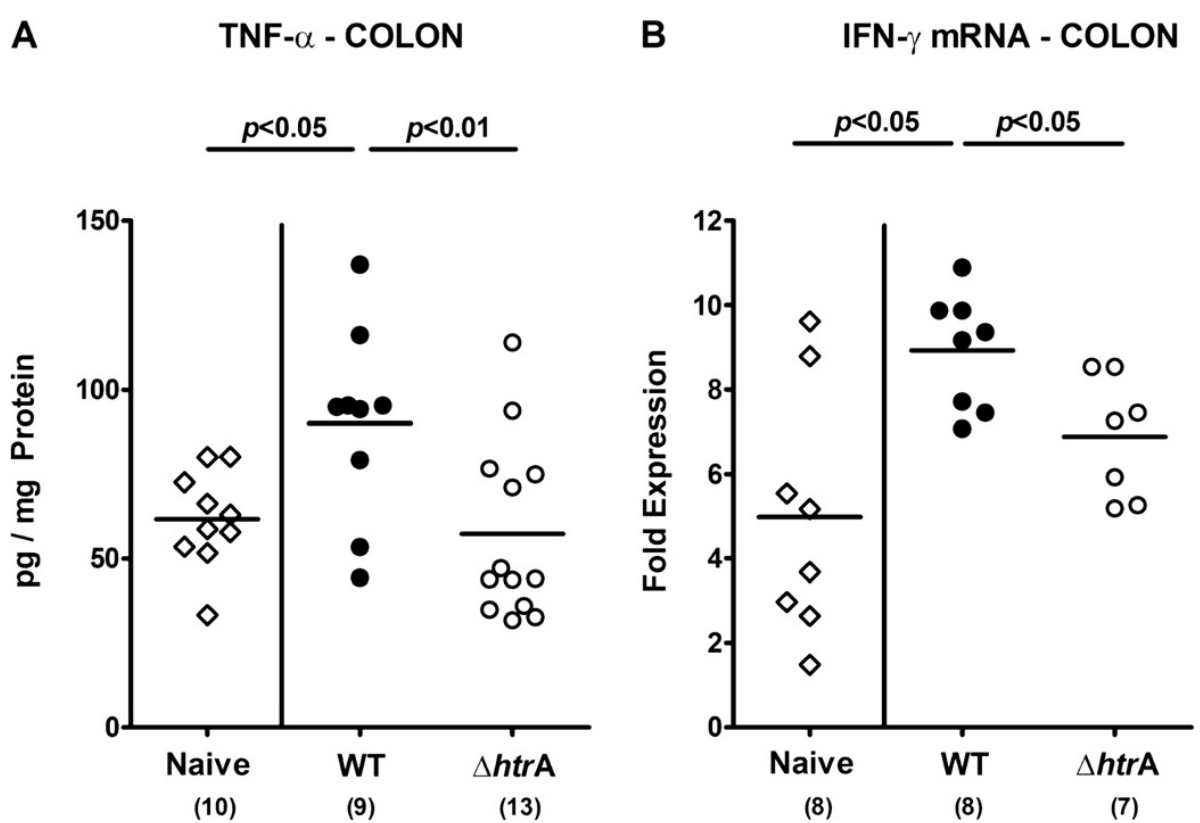

Figure 4 HtrA impacts colonic pro-inflammatory cytokine expression during $C$. jejuni infection. Immediately after weaning, infant mice

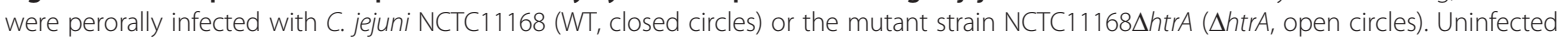
animals (Naïve; open diamonds) served as negative controls. (A) TNF-a protein and (B) IFN- $\gamma$ mRNA expression levels were determined in ex vivo biopsies taken from colons at day 7 p.i. by ELISA and quantitative real time PCR technique, respectively. Numbers of analyzed animals are given in parentheses. Means (black bars) and levels of significance ( $P$-values) determined by the Mann-Whitney- $U$ test are indicated. Data shown were pooled from at least two independent experiments.

A

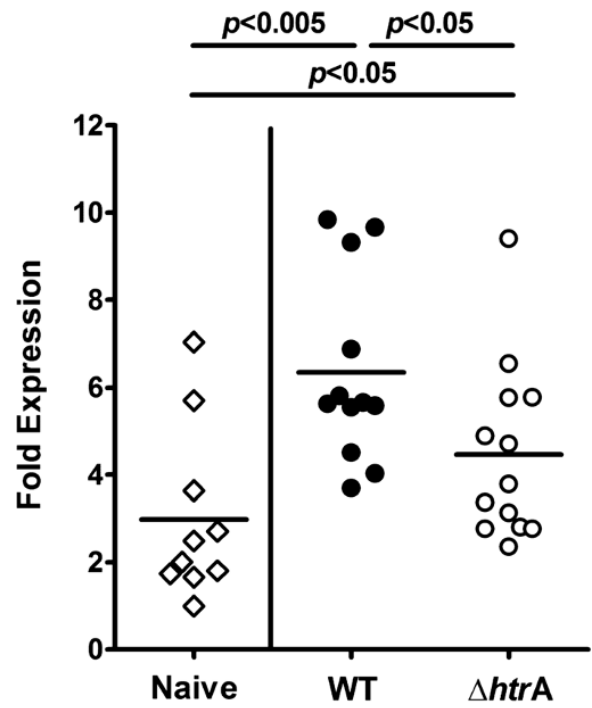

(10)

(12)

(13)

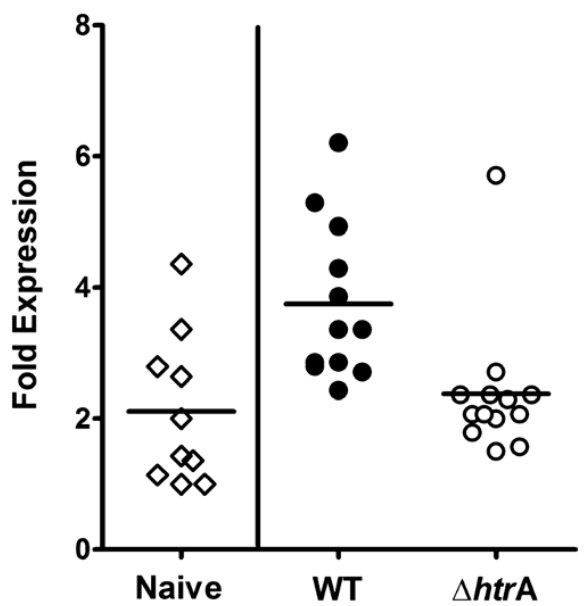

(10)

(12)

(13)

Figure 5 Colonic matrixmetalloproteinase-2 expression following C. jejuni infection of infant mice. Immediately after weaning, infant mice

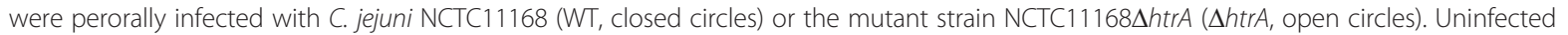
animals (Naïve; open diamonds) served as negative controls. (A) MMP-2 and (B) TIMP-1 mRNA expression levels were determined in colonic ex vivo biopsies taken at day 7 p.i. by quantitative real-time PCR. Numbers of analyzed animals are given in parentheses. Means (black bars) and levels of significance ( $P$-values) determined by the Mann-Whitney-U test are indicated. Data shown were pooled from three independent experiments. 
Given that the matrix-degrading endopeptidase matrixmetalloproteinase (MMP)-2 is upregulated during intestinal immunopathology in mice and men [32-36], we next assessed colonic expression levels of MMP-2 and its endogenous inhibitor, the tissue inhibitor of matrixmetalloproteinase (TIMP)-1. Seven days following C. jejuni infection, MMP-2 mRNA expression levels increased multi-fold, but less distinctly in colons of $\Delta h t r A$ as compared to parental strain infected mice ( $\mathrm{p}<0.05$; Figure 5A). Furthermore, colonic TIMP-1 mRNA expression was upregulated 7 days following parental but not $\Delta h t r A$ strain infection $(\mathrm{p}<0.01$; Figure $5 \mathrm{~B})$. Taken together, absence of the $h \operatorname{tr} A$ gene is associated with less distinct $C$. jejuni induced apoptosis and inflammation in the intestinal tract.

\section{C. jejuni HtrA plays a crucial role in the induction of extra-intestinal immune responses}

We have recently shown that asymptomatic longterm C. jejuni carrying mice displayed inflammatory immune responses in extra-intestinal compartments such as liver, kidneys and lungs [30]. We here investigated extra-intestinal $C$. jejuni induced sequelae as early as 7 days p.i. To address this we quantified inflammatory changes in H\&E stained paraffin sections of liver, kidneys and lungs applying respective standardized histopathological scores. Remarkably, 7 days following $C$. jejuni infection with either strain mild to moderate histopathological changes could be observed in extraintestinal organs that were exclusively lacking viable $C$. jejuni (Figure 6). Whereas only subtle inflammatory infiltrates could be observed in livers of infected infant mice irrespective of the C. jejuni strain (Figure 6A), histopathological scores for kidneys and lungs were lower in $\Delta h t r A$ mutant as compared to parental strain infected animals at day 7 p.i. (Figure 6B, C), indicative for less distinct inflammatory disease in the respective extra-intestinal organs due to $h t r A$ deficiency of the $C$. jejuni strain.

Taken together, upon $\Delta h t r A$ strain infection of infant mice large intestinal pro-inflammatory immune responses were ameliorated whereas compensatory regenerative/ proliferating properties of the epithelium were preserved. Remarkably, C. jejuni induced inflammatory sequelae in extra-intestinal organs such as liver, kidneys and lungs could be observed as early as 7 days p.i., whereas extraintestinal responses were less pronounced in the latter two compartments due to $h \operatorname{tr} A$ deficiency.

\section{Discussion}

We have recently shown in vitro that the chaperone and serine protease HtrA secreted by $C$. jejuni exerts a novel pathogenicity factor that is involved in bacterial invasion and transmigration across epithelial cells by cleaving Ecadherin and opening cell-to-cell junctions [20-23]. In the in vivo study presented here we investigated the impact of the htrA gene in pathogen-host-interaction and induction of immunopathology upon $C$. jejuni infection. To address this, conventionally colonized infant mice were infected either with the $C$. jejuni knockout mutant NCTC11168 4 trA or its syngenic parental WT strain at the age of 3 weeks immediatedly after weaning. Even though only a subset of mice harboured the respective strain in the intestinal tract, about one third of infected mice suffered from bloody diarrhea. In a previous infection study with another $C$. jejuni strain (B2), having highly efficient colonizing properties, virtually all infant mice harboured the pathogen at day 7 p.i., whereas up to $90 \%$ of mice displayed bloody diarrhea [30].
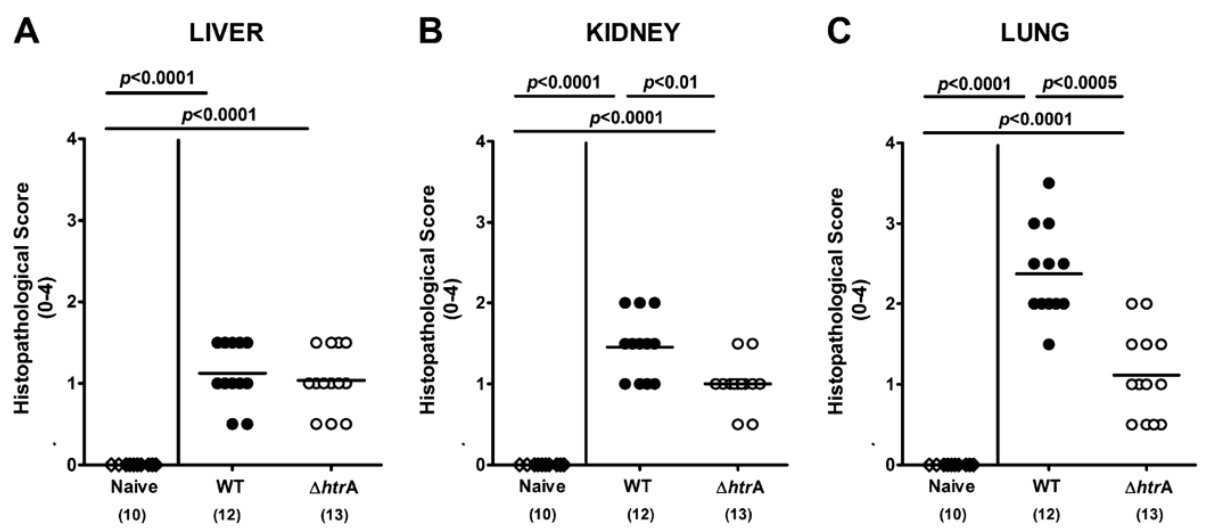

Figure 6 Role of HtrA in extra-intestinal histopathological sequelae following C. jejuni infection of infant mice. Immediately after

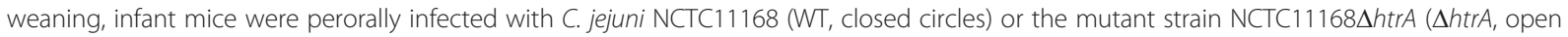
circles). Uninfected animals (Naïve; open diamonds) served as negative controls. Seven days following C. jejuni infection extra-intestinal immunopathological sequelae were assessed in H\&E stained paraffin sections taken from (A) liver, (B) kidneys, and (C) lungs by applying respective standardized histopathological scoring systems. Means (black bars), levels of significance ( $P$-values) determined by the Mann-Whitney-U test and numbers of analyzed animals (in parentheses) are indicated. Data shown were pooled from three independent experiments. 
However, in our experiments with parental strain NCTC11168, but not $\Delta h t r A$ mutant infected infant mice exhibited multi-fold increased numbers of colonic apoptotic cells at day 7 p.i. as compared to naïve controls. Conversely, the number of proliferating cells was significantly increased in $\Delta h t r A$ but not parental strain infected mice indicative for up-regulated regenerative properties of intestinal epithelial cells thereby counteracting $C$. jejuni induced tissue damage. Less pronounced intestinal immunopathology due to the absence of HtrA was further underlined by lower expression levels of colonic pro-inflammatory cytokines such as TNF- $\alpha$ and IFN- $\gamma$, which have been shown to be key cytokines mediating $C$. jejuni induced immunopathology in murine infection models with different clinical severity $[24,25]$. Interestingly, less distinct intestinal immunopathology was accompanied by lower colonic expression levels of the matrix-degrading enzyme MMP-2 and its endogenous inhibitor TIMP-1 seven days following $\triangle h t r A$ as compared to the parental strain infection. These MMP expression data are in good agreement with previous studies demonstrating that MMP-2 is up-regulated in acute and chronic small as well as large intestinal inflammation in mice and men [32,33,35-38]. For the first time we have now presented evidence that MMP-2 might also play an important role in mediating $C$. jejuni-induced disease, which is currently further unravelled in ongoing studies.

Surprisingly, rather mild to moderate histopathological sequelae of $C$. jejuni infection could be detected as early as one week in extra-intestinal organs such as liver, kidneys and lungs. All organ samples were free of viable $C$. jejuni as shown by negative cultures. In our previous study, C. jejuni B2 strain infected infant mice exhibited histopathological changes in the respective organs more than 100 days p.i. [30] with inflammatory foci consisting mainly of accumulated CD3-positive T cells [31]. Strikingly, in the present study, extra-intestinal histopathological changes in kidneys and lungs were less distinct one week following $\Delta h t r A$ as compared to parental strain infection. Hence, absence of the HtrA protein is not only associated with less pronounced intestinal but also extra-intestinal inflammation.

In humans, only very few cases of pathogen-associated disease manifestations affecting liver, lungs, heart or spleen have been reported in severely immuno-compromized patients with $C$. jejuni bacteremia [39-41]. Fauchere and coworkers showed in isolator-raised germfree mice that C. jejuni was cleared from extra-intestinal compartments such as liver and spleen and the circulation within 24 hours following infection most likely due to non-specific bactericidal factors such as phagocytes and complement [42]. Histopathological changes within extra-intestinal organs, however, were not investigated [42]. In the context with our previous observation that CD3-positive cells accumulate at extra-intestinal locations, it is tempting to speculate that potentially pro-inflammatory immune cell populations might be attracted to the extra-intestinal compartments very early following infection before the subsequent clearing of the pathogen. These immune cells might then further reside in the respective organs and explain the sterile inflammatory responses in extra-intestinal tissue sites observed 7 days p.i. as well as in asymptomatic longterm $C$. jejuni carriers more than 100 days p.i. [30,31].

\section{Conclusions}

Our in vivo study using the infant mouse infection model provides clear evidence for the importance of HtrA as a new virulence factor mediating $C$. jejuni induced intestinal as well as extra-intestinal immune responses. Thus, we describe here the first known $C$. jejuni mutant with very high motility [21], but having very low potential to trigger intestinal inflammation and bloody diarrhea as compared to WT bacteria. Future studies will further elucidate the underlying molecular mechanisms of C. jejuni-hostinteractions.

\section{Materials and methods}

\section{Ethics statement}

All animal experiments were conducted according to the European Guidelines for animal welfare (2010/63/EU) with approval of the commission for animal experiments headed by the "Landesamt für Gesundheit und Soziales" (LaGeSo, Berlin, Germany; registration numbers G0123/ 12). Animal welfare was monitored twice daily by assessment of clinical conditions.

\section{Mice and $C$. jejuni infection}

All mice were bred and maintained under specific pathogen-free (SPF) conditions in the facilities of the "Forschungseinrichtung für Experimentelle Medizin" (FEM, Charité - Universitätsmedizin, Berlin, Germany). Immediately after weaning, female 3-weeks-old C57BL/6 mice were infected orally with approximately $10^{9}$ viable CFU of the C. jejuni parental WT strain NCTC11168 or the isogenic mutant strain NCTC11168 $\Delta$ trA lacking the $h t r A$ gene $[21,22]$ by gavage in a total volume of $0.3 \mathrm{~mL}$ PBS on two consecutive days (day 0 and day 1 ).

\section{Clinical signs of $C$. jejuni infection, bloody feces}

To assess clinical signs of $C$. jejuni induced infection, the occurrence of blood in fecal samples was determined applying a standardized score ( 0 points: no blood; 2 points: microscopic detection of blood by the Guajac method using Haemoccult, Beckman Coulter/PCD, Krefeld, Germany; 4 points: overt blood visible) [25,43]. 


\section{Sampling procedures and histopathology}

Mice were sacrificed by isofluran treatment (Abbott, Germany). Tissue samples from liver, kidneys, lungs, and intestinal tract (duodenum, ileum, colon) were removed under sterile conditions. Intestinal samples from each mouse were collected in parallel for histopathological, immunohistochemical, microbiological, and immunological analyses. Immunohistopathological changes were determined in samples derived from colon, liver, kidneys and lungs that were immediately fixed in $5 \%$ formalin and embedded in paraffin. Sections $(5 \mu \mathrm{m})$ were stained with H\&E, examined by light microscopy (magnification $100 \times$ and $400 \times$ ) and histopathological changes quantitatively assessed by two independent double-blinded investigators applying respective histopathological scoring systems. In brief:

Colonic histopathology (max. 4 points; according to [44]): 0: no inflammation; 1: single isolated cell infiltrates within the mucosa, no epithelial hyperplasia; 2: mild scattered to diffuse cell infiltrates within the mucosa and submucosa; mild epithelial hyperplasia; starting loss of goblet cells; 3: cell infiltrates within mucosa, submucosa, and sometimes transmural; epithelial hyperplasia; loss of goblet cells; 4: cell infiltrates within mucosa, submucosa, and transmural; severe inflammation; loss of goblet cells, loss of crypts; ulcerations; severe epithelial hyperplasia. Hepatic histopathology (max. 9 points; modified Ishak score [45]): Lobular inflammation: 0: normal; 1: minimal inflammation (few inflammatory infiltrates); 2: mild inflammation (increased inflammatory cells, but less pyknotic necrosis); 3: moderate inflammation (marked increase in inflammatory cells and lots of pyknotic necroses); 4: severe inflammation (necrosis); 5 : severe inflammation (plus bridging necroses).

Portal inflammation: 0: normal; 1: mild inflammation $(<1 /$ 3 of portal tracts); 2: moderate inflammation (ca. 1/2 of portal tracts); 3: severe inflammation ( $>2 / 3$ of portal tracts); 4: severe inflammation (plus portal inflammation disperse into parenchyma).

Renal histopathology (max. 4 points; according to [46]): 0 : normal glomerulus; 1 : focal and mild hypercellularity (normal $=3$ per segment); 2 : multifocal and moderate hypercellularity with capillary dilatation and mild hyalinosis; 3 : diffuse hypercellularity ( $>50 \%$ of the tuft) and capillary aneurysm; 4: extensive sclerosis/crescents, tuft obliteration, collapse.

Pulmonal histopathology (max. 4 points, modified according to [47]):

0 : no inflammation; 1: perivascular cuff of inflammatory cells; 2: mild inflammation, extending throughout $<25 \%$ of the lung; 3: moderate inflammation covering 25-50\% of the lung; 4: severe inflammation involving $>50 \%$ of the lung.

\section{Immunohistochemistry}

In situ immunohistochemical analyses of $5 \mu \mathrm{m}$ thin colonic paraffin sections were performed as described previously $[24,25,30,31,48]$. Primary antibodies against cleaved caspase-3 (Asp175, Cell Signaling, USA, 1:200), Ki67 (TEC3, Dako, Denmark, 1:100), CD3 (M-20, Santa Cruz, 1:1000), and Foxp3 (FJK-16 s, eBioscience, 1:100) were used. For each animal the average number of positively stained cells within at least six high power fields (HPF, $0.287 \mathrm{~mm}^{2}$; 400× magnification) was determined microscopically by two independent double-blinded investigators.

\section{Quantitative analysis of $C$. jejuni}

At time of necropsy (day 7 p.i.) live $C$. jejuni were detected in luminal samples derived from the duodenum, ileum or colon dissolved in sterile PBS by culture as described earlier [24,31]. In brief, serial dilutions of fecal samples were streaked out on karmali agar (Oxoid, Wesel, Germany) and incubated in a microaerobic atmosphere at $37^{\circ} \mathrm{C}$ for at least 48 hours. The respective weights of luminal fecal samples were determined by the difference of the sample weights before and after asservation.

\section{Cytokine detection in colonic ex vivo biopsies}

Colonic biopsies were cut longitudinally and washed in PBS. Strips of approximately $1 \mathrm{~cm}^{2}$ colon were placed in 24-flat-bottom well culture plates (Nunc, Wiesbaden, Germany) containing $500 \mu \mathrm{L}$ serum-free RPMI 1640 medium supplemented with penicillin $(100 \mathrm{U} / \mathrm{mL})$ and streptomycin $(100 \mu \mathrm{g} / \mathrm{mL}$; PAA Laboratories). After $18 \mathrm{~h}$ at $37^{\circ} \mathrm{C}$ supernatants were tested for TNF- $\alpha$ by ELISA (BD Biosciences).

\section{Real-time PCR analysis}

RNA was isolated from colonic tissues using the RNeasy Mini Kit (Qiagen). mRNA was reversed transcribed and analysed in triplicate assays by TaqMan PCR using a sequence detection system (ABI Prism 7700; Applied Biosystems) as described previously [35,49]. For detection of murine IFN- $\gamma$, MMP-2 and TIMP-1 assays including double-fluorescent probes in combination with assays for the mouse housekeeping gene hypoxanthine phosphoribosyltransferase (HPRT) were purchased from Applied Biosystems). Expression levels were calculated relative to the HPRT expression.

\section{Antibodies and Western blotting}

C. jejuni cell pellets were lysed and proteins were separated by SDS-PAGE [50,51]. Coomassie blue staining was done as described [52]. The polyclonal rabbit $\alpha$ HtrA antibody was raised against a conserved peptide corresponding to amino acid (aa) residues 288-301: CQGDTKKAYKNQEGA. The peptide was conjugated to 
Limulus polyphemus haemocyanin carrier protein, and two rabbits each were immunized by Biogenes $\mathrm{GmbH}$ (Berlin, Germany) using standard protocols [53]. The resulting antiserum was affinity-purified and the specificity against the proteins in $C$. jejuni was confirmed by Western blotting [54,55]. Horseradish peroxidase-conjugated anti-rabbit polyvalent sheep immunoglobulin was used as secondary antibody (DAKO Denmark A/S, DK-2600 Glostrup, Denmark). Blots were developed with ECL Plus Western blot reagents (GE Healthcare, UK limited Amersham Place, UK) as described [56,57].

\section{Statistical analysis}

Mean values, medians, and levels of significance were determined using Mann-Whitney-U test. Two-sided probability $(P)$ values $\leq 0.05$ were considered significant. All experiments were repeated at least twice.

\section{Competing interests}

The authors have declared that no competing interests exist.

\section{Authors' contributions}

Conceived and designed the experiments: MMH AF MA SB SB. Performed the experiments: MMH AF MA UG. Analyzed the data: MMH AF MA AAK MB NT SB SB. Contributed reagents/ materials/ analysis tolls: UBG MB NT AAK. Wrote the paper: $\mathrm{MMH}, \mathrm{SB}, \mathrm{SB}$. All authors read and approved the final manuscript.

\section{Acknowledgements}

We thank Michaela Wattrodt, Ursula Rüschendorf, Ines Puschendorf, Alexandra Bittroff-Leben, Silvia Schulze, Gernot Reifenberger, Uwe Lohmann, and the staff of the animal research facility for excellent technical assistance, animal breeding and genotyping of mice. We are grateful to Simone Spieckermann for immunohistochemistry staining of colonic sections.

\section{Financial disclosure, grant support}

This work was supported by grants from the German Research Foundation (DFG) to UBG (GO363/12-1, CampyGerm; SFB633, TP A7), SB and AF (SFB633, TP A7), AAK (SFB633, TP Z1), MMH (SFB633, TP B6), MA and UG (SFB633, Immuco), and from the German Federal Ministery of Education and Research (BMBF) to SB (TP1.1). The work of SB, MB and NT is supported through a DFG grant (project B10 of CRC-796).

The funders had no role in study design, data collection and analysis, decision to publish, or preparation of the manuscript.

\section{Author details}

'Department of Microbiology and Hygiene, Charité - University Medicine Berlin, Campus Benjamin Franklin, Hindenburgdamm 27, D-12203 Berlin, Germany. ${ }^{2}$ Department of Biology, Division of Microbiology, Friedrich Alexander University Erlangen, Nuremberg, Germany. ${ }^{3}$ Department of Medicine I for Gastroenterology, Infectious Disease and Rheumatology / Research Center ImmunoSciences (RCIS), Charité - University Medicine Berlin, Berlin, Germany.

Received: 8 March 2014 Accepted: 19 May 2014 Published: 27 May 2014

\section{References}

1. Friedman CR, Neimann J, Wegener HC, Tauxe RV: Epidemiology of Campylobacter jejuni infections in the United States and other industrialized nations. In Campylobacter. Edited by Nachamkin I, Blaser MJ. Washington, DC: ASM Press; 2000:121-138.

2. Young KT, Davis LM, Dirita VJ: Campylobacter jejuni: molecular biology and pathogenesis. Nature Rev Microbiol 2007, 5:665-679.

3. Kist M, Bereswill S: Campylobacter jejuni. Contrib Microbiol 2001, 8:150-165.
4. van Spreeuwel JP, Duursma GC, Meijer CJ, Bax R, Rosekrans PC, Lindeman J: Campylobacter colitis: histological immunohistochemical and ultrastructural findings. Gut 1985, 26:945-951

5. Walker RI, Caldwell MB, Lee EC, Guerry P, Trust TJ, Ruiz-Palacios GM: Pathophysiology of Campylobacter enteritis. Microbiol Rev 1986, 50:81-94.

6. Janssen R, Krogfelt KA, Cawthraw SA, van Pelt W, Wagenaar JA, Owen RJ: Host-pathogen interactions in Campylobacter infections: the host perspective. Clin Microbiol Rev 2008, 21:505-518.

7. Allos BM: Association between Campylobacter infection and Guillain-Barré syndrome. J Infect Dis 1997, 176(Suppl 2):S125-S128.

8. ÓCróinín T, Backert S: Host epithelial cell invasion by Campylobacter jejuni: trigger or zipper mechanism? Front Cell Infect Microbiol 2012, 2:25.

9. Pei Z, Burucoa C, Grignon B, Baqar S, Huang XZ, Kopecko DJ, Bourgeois AL, Fauchere $J$, Blaser MJ: Mutation in the peb1A locus of Campylobacter jejuni reduces interactions with epithelial cells and intestinal colonization of mice. Infect Immun 1998, 66:938-943.

10. Konkel ME, Monteville MR, Rivera-Amill V, Joens LA: The pathogenesis of Campylobacter jejuni-mediated enteritis. Curr Issues Intest Microbiol 2001, 2:55-71.

11. Poly F, Guerry P: Pathogenesis of Campylobacter. Curr Opin Gastroenterol 2008, 24:27-31.

12. Novik V, Hofreuter D, Galán JE: Identification of Campylobacter jejuni genes involved in its interaction with epithelial cells. Infect Immun 2010, 78:3540-3553

13. Eucker TP, Konkel ME: The cooperative action of bacterial fibronectin-binding proteins and secreted proteins promote maximal Campylobacter jejuni invasion of host cells by stimulating membrane ruffling. Cell Microbiol 2012 14:226-238.

14. Krause-Gruszczynska M, Rohde M, Hartig R, Genth H, Schmidt G, Keo T, Koenig W, Miller WG, Konkel ME, Backert S: Role of the small Rho GTPases Rac1 and Cdc42 in host cell invasion of Campylobacter jejuni. Cell Microbiol 2007, 9:2431-2444

15. Krause-Gruszczynska M, Boehm M, Rohde M, Tegtmeyer N, Takahashi S, Buday L, Oyarzabal OA, Backert S: The signaling pathway of Campylobacter jejuni-induced Cdc42 activation: Role of fibronectin, integrin beta1, tyrosine kinases and guanine exchange factor Vav2. Cell Commun Signal 2011, 9:32

16. Boehm M, Krause-Gruszczynska M, Rohde M, Tegtmeyer N, Takahashi S, Oyarzabal OA, Backert S: Major host factors involved in epithelial cell invasion of Campylobacter jejuni: role of fibronectin, integrin beta1, FAK, Tiam-1, DOCK180 in activating Rho GTPase Rac1. Front Cell Infect Microbiol 2011, 1:17.

17. Backert S, Boehm M, Wessler S, Tegtmeyer N: Transmigration route of Campylobacter jejuni across polarized intestinal epithelial cells: paracellular, transcellular or both? Cell Commun Signal 2013, 11:72.

18. Brøndsted L, Andersen MT, Parker M, Jørgensen K, Ingmer H: The HtrA protease of Campylobacter jejuni is required for heat and oxygen tolerance and for optimal interaction with human epithelial cells. Appl Environ Microbiol 2007, 71:3205-3212.

19. Bæk KT, Vegge CS, Brøndsted L: HtrA chaperone activity contributes to host cell binding in Campylobacter jejuni. Gut Pathog 2011, 3:13.

20. Hoy B, Geppert T, Boehm M, Reisen F, Plattner P, Gadermaier G, Sewald N, Ferreira F, Briza P, Schneider G, Backert S, Wessler S: Distinct roles of secreted HtrA proteases from Gram-negative pathogens in cleaving the junctional protein and tumor suppressor E-cadherin. J Biol Chem 2012, 287:10115-10120.

21. Boehm M, Hoy B, Rohde M, Tegtmeyer N, Baek KT, Oyarzabal OA, Brondsted L, Wessler S, Backert S: Rapid paracellular transmigration of Campylobacter jejuni across polarized epithelial cells without affecting TER: role of proteolytic-active HtrA cleaving E-cadherin but not fibronectin. Gut Pathog 2012, 4:3

22. Hoy B, Löwer M, Weydig C, Carra G, Tegtmeyer N, Geppert T, Schröder P, Sewald N, Backert S, Schneider G, Wessler S: Helicobacter pylori HtrA is a new secreted virulence factor that cleaves E-cadherin to disrupt intercellular adhesion. EMBO Rep 2010, 11:798-804.

23. Boehm M, Haenel I, Hoy B, Brøndsted L, Smith TG, Hoover T, Wessler S, Tegtmeyer N: Extracellular secretion of protease HtrA from Campylobacter jejuni is highly efficient and independent of its protease activity and flagellum. Europ J Immunol Microbiol 2013, 3:163-173.

24. Bereswill S, Fischer A, Plickert R, Haag LM, Otto B, Kühl AA, Dasti JI, Zautner AE, Muñoz M, Loddenkemper C, Gross U, Göbel UB, Heimesaat MM: Novel 
murine infection models provide deep insights into the "Menage a Trois" of Campylobacter jejuni, microbiota and host innate immunity. PLOS ONE 2011, 6:e20953.

25. Haag LM, Fischer A, Otto B, Plickert R, Kühl AA, Göbel UB, Bereswill S, Heimesaat MM: Campylobacter jejuni induces acute enterocolitis in gnotobiotic IL-10-/- mice via Toll-like-receptor-2 and -4 signaling. PLOS ONE 2012, 7:e40761

26. Diker KS, Hascelik G, Diker S: Colonization of infant mice with flagellar variants of Campylobacter jejuni. Acta Microbiol Hung 1992, 39:133-136.

27. Hänninen ML: Bismuth subsalicylate in the prevention of colonization of infant mice with Campylobacter jejuni. Epidemiol Infect 1990, 104:397-404.

28. Abimiku AG, Dolby JM: The mechanism of protection of infant mice from intestinal colonisation with Campylobacter jejuni. J Med Microbiol 1987 23:339-344.

29. Field LH, Pope LM, Cole GT, Guentzel MN, Berry LJ: Persistence and spread of Candida albicans after intragastric inoculation of infant mice. Infect Immun 1981, 31:783-791.

30. Haag LM, Fischer A, Otto B, Grundmann U, Kühl AA, Göbel UB, Bereswill S, Heimesaat MM: Campylobacter jejuni infection of infant mice: acute enterocolitis is followed by asymptomatic intestinal and extra-intestinal immune response. Eur J Microbiol Immunol 2012, 2:2-11.

31. Heimesaat MM, Haag LM, Fischer A, Otto B, Kühl AA, Göbel UB, Bereswill S: Survey of extra-intestinal immune responses in asymptomatic long-term Campylobacter jejuni-infected mice. Eur J Microbiol Immunol 2013, 3:174-182

32. Bailey CJ, Hembry RM, Alexander A, Irving MH, Grant ME, Shuttleworth CA: Distribution of the matrix metalloproteinases stromelysin, gelatinases $A$ and B, and collagenase in Crohn's disease and normal intestine. J Clin Pathol 1994, 47:113-116.

33. Baugh MD, Perry MJ, Hollander AP, Davies DR, Cross SS, Lobo AJ, Taylor CJ, Evans GS: Matrix metalloproteinase levels are elevated in inflammatory bowel diseases. Gastroenterol 1999, 117:814-822.

34. Salmela MT, MacDonald TT, Black D, Irvine B, Zhuma T, Saarialho-Kere U, Pender SL: Upregulation of matrix metalloproteinases in a model of $T$ cell mediated tissue injury in the gut: analysis by gene array and in situ hybridisation. Gut 2002, 51:540-547.

35. Muñoz M, Heimesaat MM, Danker K, Struck D, Lohmann U, Plickert R, Bereswill S, Fischer A, Dunay IR, Wolk K, Loddenkemper C, Krell HW, Libert C, Lund LR, Frey O, Hölscher C, Iwakura Y, Ghilardi N, Ouyang W, Kamradt T, Sabat R, Liesenfeld O: Interleukin (IL)-23 mediates Toxoplasma gondiiinduced immunopathology in the gut via matrixmetalloproteinase- 2 and IL-22 but independent of IL-17. J Exp Med 2009, 206:3047-3059.

36. Heimesaat MM, Dunay IR, Fuchs D, Trautmann D, Fischer A, Kühl AA, Loddenkemper C, Siegmund B, Batra A, Bereswill S, Liesenfeld O: The distinct roles of MMP-2 and MMP-9 in acute DSS colitis. Eur J Microbiol Immunol 2011, 1:302-310.

37. Heuschkel RB, MacDonald TT, Monteleone G, Bajaj-Elliott M, Smith JA, Pender SL: Imbalance of stromelysin-1 and TIMP-1 in the mucosal lesions of children with inflammatory bowel disease. Gut 2000, 47:57-62.

38. von Lampe B, Barthel B, Coupland SE, Riecken EO, Rosewicz S: Differential expression of matrix metalloproteinases and their tissue inhibitors in colon mucosa of patients with inflammatory bowel disease. Gut 2000, 47:63-73.

39. Pigrau C, Bartolome R, Almirante B, Planes AM, Gavalda J, Pahissa A: Bacteremia due to Campylobacter species: clinical findings and antimicrobial susceptibility patterns. Clin Infect Dis 1997, 25:1414-1420.

40. Tee W, Mijch A: Campylobacter jejuni bacteremia in human immunodeficiency virus (HIV)-infected and non-HIV-infected patients: comparison of clinical features and review. Clin Infect Dis 1998, 26:91-96.

41. Crushell E, Harty S, Sharif F, Bourke B: Enteric campylobacter: purging its secrets? Pediatr Res 2004, 55:3-12.

42. Fauchère $J$, Véron $M$, Lellouch-Tubiana $A$, Pfister $A$ : Experimental infection of gnotobiotic mice with Campylobacter jejuni: colonisation of intestine and spread to lymphoid and reticulo-endothelial organs. J Med Microbiol 1985, 20:215-224.

43. Heimesaat MM, Fischer A, Siegmund B, Kupz A, Niebergall J, Fuchs D, Jahn HK, Freudenberg M, Loddenkemper C, Batra A, Lehr HA, Liesenfeld O, Blaut M, Göbel UB, Schumann RR, Bereswill S: Shift towards pro-inflammatory intestinal bacteria aggravates acute murine colitis via Toll-like receptors 2 and 4. PLOS ONE 2007, 2:e662.

44. Paclik D, Berndt U, Guzy C, Dankof A, Danese S, Holzloehner P, Rosewicz S, Wiedenmann B, Wittig BM, Dignass AU, Sturm A: Galectin-2 induces apoptosis of lamina propria T lymphocytes and ameliorates acute and chronic experimental colitis in mice. J Mol Med 2008, 86:1395-1406.

45. Ishak K, Babtista A, Bianchi L, Callea F, De Groote J, Gudat F, Denk H, Desmet V, Korb G, MacSween RNM, Philipps MJ, Portmann BG, Poulsen H, Scheuer PJ, Schmid M, Thaler H: Histological grading and staging of chronic hepatitis. J Hepatol 1995, 22:696-699.

46. Appel GB, Silva FG, Pirani CL, Meltzer Jl, Estes D: Renal involvement in systemic lupus erythematosus (SLE): a study of 56 patients emphasizing histologic classification. Medicine 1978, 57:371-410.

47. Blok DC, van der Sluijs KF, Florquin S, de Boer OJ, van 't Veer C, de Vos AF, van der Poll T: Limited anti-inflammatory role for interleukin-1 receptor like 1 (ST2) in the host response to murine postinfluenza pneumococcal pneumonia. PLOS ONE 2013, 8:e58191.

48. Heimesaat MM, Nogai A, Bereswill S, Plickert R, Fischer A, Loddenkemper C, Steinhoff U, Tchaptchet S, Thiel E, Freudenberg MA, Göbel UB, Uharek L. MyD88/TLR9 mediated immunopathology and gut microbiota dynamics in a novel murine model of intestinal graft-versus-host disease. Gut 2010, 59:1079-1087

49. Wolk DM, Schneider SK, Wengenack NL, Sloan LM, Rosenblatt JE: Real-time PCR method for detection of Encephalitozoon intestinalis from stool specimens. J Clin Microbiol 2002, 40:3922-3928.

50. Wiedemann T, Hofbaur S, Tegtmeyer N, Huber S, Sewald N, Wessler S, Backert S, Rieder G: Helicobacter pylori CagL dependent induction of gastrin expression via a novel av $\beta 5$-integrin-integrin linked kinase signalling complex. Gut 2012, 61:986-996.

51. Mueller D, Tegtmeyer N, Brandt S, Yamaoka Y, De Poire E, Sgouras D, Wessler S, Torres J, Smolka A, Backert S: c-Src and c-Abl kinases control hierarchic phosphorylation and function of the CagA effector protein in Western and East Asian Helicobacter pylori strains. J Clin Invest 2012, 122:1553-1566.

52. Hirsch C, Tegtmeyer N, Rohde M, Rowland M, Oyarzabal OA, Backert S: Live Helicobacter pylori in the root canal of endodontic-infected deciduous teeth. J Gastroentero/ 2012, 47:936-940.

53. Tegtmeyer N, Rivas Traverso F, Rohde M, Oyarzabal OA, Lehn N, Schneider-Brachert W, Ferrero RL, Fox JG, Berg DE, Backert S: Electron microscopic, genetic and protein expression analyses of Helicobacter acinonychis strains from a Bengal tiger. PLOS ONE 2013, 8:e71220.

54. Tegtmeyer N, Wittelsberger R, Hartig R, Wessler S, Martinez-Quiles N, Backert S: Serine phosphorylation of cortactin controls focal adhesion kinase activity and cell scattering induced by Helicobacter pylori. Cell Host Microbe 2011, 9:520-531.

55. Backert S, Hofreuter D: Molecular methods to investigate adhesion, transmigration, invasion and intracellular survival of the foodborne pathogen Campylobacter jejuni. J Microbiol Meth 2013, 95:8-23.

56. Conradi J, Tegtmeyer N, Woźna M, Wissbrock M, Michalek C, Gagell C, Cover $T L$, Frank R, Sewald N, Backert S: An RGD helper sequence in CagL of Helicobacter pylori assists in interactions with integrins and injection of CagA. Front Cell Infect Microbiol 2012, 2:70.

57. Kim DJ, Park JH, Franchi L, Backert S, Núñez G: The Cag pathogenicity island and interaction between TLR2/NOD2 and NLRP3 regulate IL-1 $\beta$ production in Helicobacter pylori infected dendritic cells. Eur J Immunol 2013, 43:2650-2658.

doi:10.1186/1757-4749-6-16

Cite this article as: Heimesaat et al:: The impact of serine protease HtrA in apoptosis, intestinal immune responses and extra-intestinal histopathology during Campylobacter jejuni infection of infant mice. Gut Pathogens 2014 6:16. 